\title{
Examining learning in relation to the contexts of use of ICT
}

\author{
A. Tolmie \\ Department of Psychology, University of Strathclyde
}

Correspondence:

Dr Andrew Tolmie,

Department of Psychology,

University of Strathclyde,

40 George Street,

Glasgow G1 1QE

email: a.k.tolmie@strath.ac.uk 


\title{
Examining learning in relation to the contexts of use of ICT
}

\begin{abstract}
Although ICT resources are commonly expected to produce uniform benefits, they are necessarily employed within pre-existing contexts of educational and social activity, and the outcome in terms of both pattern of use and learning depends on how they fit in with these. As a result, the same technology or software may have unexpectedly diverse effects, according to specific setting. If the object is to exercise control over outcome, then the conditions of use need to be planned for within the design and implementation of the technology. In order to do this, it is crucial that research gathers data on how outcomes are affected by the interplay between technology and context. This raises questions about the methods that would be appropriate for the conduct and dissemination of such research. These points are discussed in relation to three studies, one each at primary, secondary and university levels of education.
\end{abstract}

Keywords: context effects, evaluation, educational context, social context, measurement of fit, dissemination 
It is a longstanding complaint that policy-makers, administrators and funding agencies typically look for direct and obvious benefits from the introduction of ICT into education, despite the fact that the complexities of the educational process make it unlikely that any new element could have a straightforward impact (see e.g. Crook, 1994). In the UK at least, this oversimplified approach has arguably spread to developers, as a result of the Higher Education Funding Councils' Teaching and Learning Technologies Programme. This programme has administered a large proportion of the funding available for the development of educational ICT over the last decade, but because it has mostly been concerned with tackling circumscribed needs, it has encouraged a mentality of one-stop resources and limited evaluations of the effectiveness of those resources (see Oliver \& Conole, 1998).

Thus developers commonly see it as appropriate to aim for blanket take-up of a particular ICT provision, in the expectation of fairly uniform outcomes. Resistance to take-up is disparagingly tagged as a product of the "not invented here syndrome" (see e.g. Robinson, Smith, Galpin, Birchall \& Turner, 1998), rather than of valid concerns about differences between contexts. This stance is reinforced by the tendency of evaluations to focus on the use of technology and its impact on learning in isolation; i.e. ignoring the broader educational activity of which it forms a part, how that either constrains use of the technology or changes as a result of its introduction, and with what effects. Evaluation work also rarely does more than examine the explicitly intended effects of ICT, and so fails to identify unintended or serendipitous repercussions that may actually be a critical aspect of its impact (Jones, 1998).

The result of this narrow perspective is that ICT resources, when brought into actual practice, frequently appear to have puzzlingly variable or disappointing outcomes, something which is often attributed to teachers possessing inadequate IT skills or having undertaken inadequate preparation (Crook, 1994). This pattern strengthens the position of 'techno-sceptics' who argue that the evidence for ICT having real benefits is limited (see Underwood, 2000). This verdict may in fact be unnecessarily pessimistic, but to challenge it, a more sophisticated, context-sensitive approach needs to be adopted both to the introduction and the evaluation of ICT in education.

\section{The effects of context}

Whatever form they take, ICT resources are necessarily introduced into a pre-existing framework of educational and social activity, within which teachers and students are used to doing things in certain ways. Since the familiarity of this framework lends it considerable inertia, how any given resource is used, and with what consequences for 
learning, depends inevitably on how it fits in with these established patterns of activity. The same technology or software may thus have unexpectedly diverse effects, according to the specific setting in which it is employed.

An illustration of this is provided by research reported in Tolmie \& Howe (1993). This study looked at secondary school pupils aged between 12 and 15 years working in pairs on physics software that dealt with the trajectories of falling objects. These pairs, of whom more or less equal numbers were female (FF), male (MM) and mixed (FM), were presented with a series of problems on-screen. The objective in each case was to agree a prediction about the path a falling object would take, input this to the computer via on-screen markers, compare it with an overlaid image of the correct solution, and account for any discrepancies between the two. It was hypothesised that use of the software would, under certain conditions, generate dialogue (especially attempts to explain predictions and solutions) that would promote improved understanding of the underlying physical principles.

In order to investigate this, the research examined how far the learning that took place (as measured by change in individual pupils' performance on a post-test relative to that on a pre-test) was associated with the incidence of different types of dialogue (predictions, disagreements, and explanations). As anticipated, learning was found to be variable in extent, as was the incidence of "productive" dialogue. A proportion of this variability was attributable to the degree of difference between individuals' original conceptions of what influenced object path, the mechanism being tested by the study (see Piaget, 1985 and Doise \& Mugny, 1984, on the effects of sociocognitive conflict). The more members of a pair differed in this respect, the more they disagreed and had to explain and justify their positions, and the greater the resultant learning.

Beyond this, though, there was a marked effect of gender pairing, with different combinations showing systematically different sequences of dialogue and influences on learning. For MM pairs, for instance, initial differences over the predicted path on a particular problem led to a discussion of the factors at work in that specific example, which was used to direct the input of a suggested solution. The feedback provided by the correct answer was then used to reappraise the role of the posited factors. This process tended to produce improved solutions, with knock-on effects on learning. In contrast, for FF pairs, differences in predictions and explanations were dealt with by simply reiterating previous responses and building up an "acceptable" account; i.e. by avoiding dealing with discrepancies in explanations, or between suggested solution 
and feedback, and focusing instead on consensual ideas. Learning appeared to be a function of the resulting unsettling of conceptions and improved individual insight. For FM pairs, differences between individuals over predictions and explanations also led to an avoidance strategy, except that here it took the form of ceding control for the input of predictions on a rigid turn and turn about basis. The level of dialogue was substantially below that in the MM and FF pairs, and learning seemed to be a function of private reactions to on-screen responses.

The most plausible interpretation of these effects is that the female and male pupils participating in this study possessed well-established patterns of interaction (or noninteraction!) with each other, especially with respect to conflict management. For males working together, this involved focusing on the concrete aspects of the problem in hand. For females, it took the form of a focus on consensus. For males and females together, the strategy was apparently to avoid discussion altogether, if possible. It would appear that these established patterns shaped pupils' use of the software, and consequently the learning processes and outcomes that were manifested, according to the combination involved. These results carry a more general implication, however: if variations of this kind occur as a function of gender, they are also likely to arise as a function of other social and cultural determinants of interactional style and computer usage. In other words, context effects will be the norm rather than the exception.

\section{Examining context effects}

Since the ability to exercise control over learning outcomes is usually a central concern with regard to the introduction of ICT resources, these context effects are of no little significance. In particular, they suggest that the influences arising from the conditions under which given resources are used need to be taken into account and managed by either the design of the technology or its implementation. To do this, though, research and evaluation work need to gather more systematic data than is usual on:

1) the interplay between technology and context, and how the former fits in with the latter under different circumstances;

2) the effects this has on how technology is used, and conversely how other activities change as a result of its introduction;

3) the consequences (unintended as well as intended) of this interplay for learning. In other words, the whole implementation 'event' must be examined, not just the use of the technology in isolation. 
This raises questions about appropriate methods for conducting such research. A full examination of the issues involved is not possible here, but one central point is that data should ideally be collected from actual use in real contexts; laboratory work will tend to be silent on too many issues of importance. The kind of approach that might be productive for investigating the fit of technology to other activity within real contexts is illustrated by Tolmie \& Boyle (2000). This research looked at the use of a computer conferencing resource for preparation of joint seminar papers, amongst other things, by a cohort of postgraduate Educational Psychology students, who spent much of each week on professional placements in different locations. These students were split into two groups for their collaborative work, and one of the main points to emerge from the research was that these groups differed markedly in the extent of their use of the conferencing resource. For instance, in one (Group 1), four of the six group members made regular use of the system over a number of weeks in the preparation of their joint seminar paper. In the other (Group 2), only two did so, and then only briefly.

The key question was how and why such differences had arisen between students with ostensibly very similar characteristics. One possibility was that Group 2 had failed to engage with the task to the same extent. However, this was not in fact the pattern revealed by one evaluation tool, a log of contact and activity, which was specifically designed to examine how use of the technology had fitted in with other activity. These logs consisted of proforma sheets which students were asked to complete during work on the preparation of their seminar paper. Entries consisted of brief details of each activity that was relevant to the task, how long this took, who else was involved (if anyone), and the medium used for any communication.

What the logs revealed was that the two groups adopted quite different working practices (see Table 1). Members of Group 1, for example, spent roughly the same amount of time on face-to-face meetings and computer conferencing, made little use of the telephone, and devoted a large proportion of their time to independent activity, such as accessing information and preparing draft sections of the seminar paper. Faceto-face communication took place at points of negotiation, whilst email was used for the exchange of drafts, and for updating others on progress. For members of Group 2, the total time devoted to the seminar task was similar to Group 1, but they spent proportionately much less on conferencing, and more on face-to-face and fax communication. Moreover, for these students, face-to-face interaction included working together, as well as the strategic activity engaged in by Group 1. 
Thus the lack of email contact between members of Group 2 did not signify a lack of engagement in the task, but a different fit of the technology to their overall pattern of activity. A subsequent questionnaire revealed that there was greater concern in this group about the reliability of the conferencing resource, based on problems they had encountered at the start-up stage. They had consequently taken a decision to split into pairs and handle the work through "tried and true" methods of face-to-face and fax or telephone contact. It is worth noting the importance once again of the past history of students' interactions as an influence on their use of technology. It is also worth stressing the value of the log of contact and activity in establishing the meaning of a pattern of online interaction that would otherwise have been obscure or liable to misinterpretation.

\section{Context effects and unintended consequences for learning}

Higher Education students, especially at postgraduate level, are likely to have the freedom and the competence to decide for themselves how to embed technology into their activity in a way that primary or secondary school pupils do not. There is thus greater potential for them to exhibit variability. However, even if student use of technology takes place under the guidance of teachers, pre-existing activity is still likely to shape both use and outcome. Rather than simply seeing such effects as 'noise' to be controlled, though, it is important to recognise that they may serve to enrich the impact of the technology, and provide opportunities to be capitalised upon. The need to be sensitive to unintended processes and consequences stemming from the introduction of ICT resources has already been noted. The reason for this is that it is possible for a resource to be successful in unexpected ways because the context in which it is put allows students to squeeze more out of the experience than anticipated.

An illustration of this is detailed by Tolmie, Howe, Duchak-Tanner \& Rattray (1999). This research examined the effectiveness of software designed to support learning about "fair testing" (i.e. controlled experimentation) in science amongst 9 to 14 year olds. Children worked in threesomes on a collaborative hypothesis testing task relating to shadow formation. This task required them to agree hypotheses about whether or not each of five factors (e.g. lamp brightness and object position) affected shadow size, and then select one hypothesis to test using a set of physical equipment 
that had been provided for the purpose. This equipment consisted of a screen and rack arrangement which allowed three different settings to be chosen for each factor, separately or in combination. Decisions about the conduct of tests, especially which settings to use, were input to a computer positioned alongside the apparatus. A "supported" version of the software monitored children's decisions, and issued warning prompts if the settings departed from a fair test (i.e. one where only the factor being tested was manipulated, and all other factors were kept constant). The impact of this software was assessed by comparison to that of an "unsupported" version identical in all respects save for the issuing of prompts. Computer and video records were used to gauge groups' on-task performance, and individual learning was measured by pre- to post-test change.

Insert Table 2 about here

The research produced a surprising result. As far as on-task performance was concerned, groups who worked with the supported software did much better than those who worked with the unsupported, conducting significantly more fair tests (see Table 2). However, the benefits of this did not apparently feed through to learning, since the two conditions produced levels of pre- to post-test change that were not significantly different from each other. It is important to note that the implication of these results was not that the supported software was ineffective, but that the children who used the unsupported version learnt more than expected. What was less apparent was how this had come about. However, one other revealing piece of information was provided by children's responses to questions posed to them at the end of the group task, about the principles of fair testing. The responses of those who had used the supported software were significantly better, in line with their on-task performance, suggesting that they had acquired an explicit grasp of fair testing as a result of the prompts issued by the software. Children in the unsupported groups had failed to acquire this grasp, but they nevertheless made up ground by the time of the post-test a few weeks later.

Since there were no signs that children engaged in independent research post-task, it would seem the explanation must be that in the absence of the software prompts, the group task still provided implicit cues to the nature of fair testing procedures, which children managed to flesh out during post-task reflection (see Piaget, 1985). The 
obvious candidate for the source of these cues appeared to be the task materials, and especially a grid of factors and levels presented by the software for selecting test settings, which implied that the factors were to be thought of separately from each other. However, the cues provided by this grid and by the rest of the task were relatively subtle (sufficiently so that they were overlooked by the researchers at first). If these were the source of the learning shown by children in the unsupported groups, this suggests that the children were particularly primed to notice them. This may be unsurprising, though, given that the activity was set in the context of a "science lesson", with all the connotations this carries of doing things systematically, something with which the grid of factors might be likely to resonate quite strongly.

\section{Conclusions: the role of dissemination}

What has been presented here is a necessarily limited range of examples, but these serve to illustrate something of why there is a need to adopt a context-sensitive approach to both the introduction and the evaluation of ICT in education, in order to discern and subsequently manage its real impact. Hopefully, these examples also provide an indication of the types of issue it might be important to examine when considering the fit between technology and its context of use, and the effects this has on learning.

It has been less possible to address the question of how information from contextsensitive evaluations might feed into the design or revision of ICT resources, in part because of the lack of sufficient instances from which to draw out general principles. There is a further difficulty that needs to be acknowledged, however. This is that it would appear likely that the range of potential context effects surrounding any given resource will be of such diversity that it may be impossible (or nearly so) for these to be adequately managed within any single software design. Thus any management effort must inevitably be located less within the design and more within the process of implementation.

This implies in turn a radical change in the philosophy underpinning the evaluation of ICT. The purpose of context-sensitive evaluations cannot be so much to inform developers, as to inform users as to the type of effects they may need to take into account when introducing a resource, and how it might be possible to deal with or capitalise upon these. Thus dissemination, and how this is approached must necessarily become a central facet of effective evaluation. There are a host of issues here that have barely been considered as yet, such as how to present such information in a form that teachers can readily understand and use. "Grounded guidelines" (see 
McAteer, Crook, Tolmie, Macleod, Musselbrook \& Barrowcliff, 2000) which provide general messages from context-oriented research alongside concrete illustrations and commentaries from real implementations may be one way forward, but much remains to be done.

\section{Acknowledgements}

A version of this paper was first presented at the British Psychological Society Education Section Annual Conference, University of Greenwich, November 1999.

The helpful comments received on that occasion are gratefully acknowledged.

\section{References}

Crook, C. (1994). Computers and the Collaborative Experience of Learning. London: Routledge.

Doise, W. \& Mugny, G. (1984). The Social Development of the Intellect. Oxford: Pergamon.

Jones, C. (1998). Evaluating a colaborative online learning environment. Active Learning, 9, 31-35.

McAteer, E., Crook, C., Tolmie, A., Macleod, H., Musselbrook, K. \& Barrowcliff, D. (2000). Learning Networks/Communication Skills.

http://www.gla.ac.uk/lncs/index.htm

Oliver, M. \& Conole, G. (1998). Evaluating communication and information technologies: a toolkit for practitioners. Active Learning, 8, 3-8.

Piaget, J. (1985). The Equilibration of Cognitive Structures. Chicago: University of Chicago Press.

Robinson, H., Smith, M., Galpin, F., Birchall, D. \& Turner, I. (1998). As good as IT gets: have we reached the limits of what technology can do for us? Active Learning, 9, 50-53.

Tolmie, A. \& Boyle, J. (2000). Factors influencing the success of computer mediated communication (CMC) environments in university teaching: a review and case study. Computers and Education, 34, 119-140. 
Tolmie, A. \& Howe, C.J. (1993). Gender and dialogue in secondary school physics. Gender \& Education, 5, 191-209.

Tolmie, A., Howe, C.J., Duchak-Tanner, V. \& Rattray, C. (1999). Explicit and implicit support for procedural learning within collaborative group work in science. IXth European Conference on Developmental Psychology, Spetses, Greece.

Underwood, J. (2000). Learning in the digital age: are teachers the key to success? Psychology of Education Review, 24, 3-7. 
Table 1. Average time (in minutes) spent by members of Group 1 and Group 2 on different activities whilst working on the seminar task (Tolmie \& Boyle, 2000).

\section{Activity}

Face-to-face communication

Email communication

Telephone communication

Independent activity

\section{Group 1}

119

97

12

281

\section{Group 2}

200

21

29

242 
Table 2: Mean number (percentage) of fair tests conducted on task by supported and unsupported groups, mean pre- to post-test change (scale of 0 to 4), and mean score on principles of fair testing (scale of 0 to 2) (Tolmie et al.,1999).

$\begin{array}{llcc} & \text { Fair Tests } & \text { Pre/Post } & \text { Principles } \\ \text { Supported } & 3.54(96 \%) & +1.84 & 1.45 \\ \text { Unsupported } & 1.56(44 \%) & +1.36 & 1.04\end{array}$

